Full length article

\title{
Comparable walking gait performance during executive and non-executive cognitive dual-tasks in chronic stroke: A pilot study
}

\author{
Elizabeth A. Walshe ${ }^{\mathrm{a}, \mathrm{b}, \mathrm{c}, *}$, Richard A.P. Roche ${ }^{\mathrm{a}}$, Christina Ward ${ }^{\mathrm{a}}$, Matt Patterson ${ }^{\mathrm{d}}$, \\ Desmond O’Neill $^{\mathrm{a}, \mathrm{e}}$, Ronan Collins ${ }^{\mathrm{e}}$, Seán Commins ${ }^{\mathrm{a}}$ \\ ${ }^{a}$ Maynooth University, Maynooth, Co Kildare, Ireland \\ ${ }^{\mathrm{b}}$ Annenberg Public Policy Center, University of Pennsylvania, Philadelphia, PA, USA

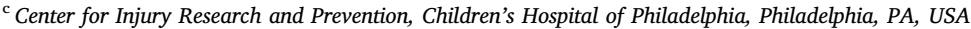 \\ ${ }^{\mathrm{d}}$ Insight Center for Data Analytics, University College Dublin, Dublin, Ireland \\ e Tallaght University Hospital, Dublin, Ireland
}

A R T I C L E I N F O

\section{Keywords:}

Gait

Stroke

Dual-task

Executive function

Older adults

\begin{abstract}
A B S T R A C T
Background: Falls are a serious problem among stroke survivors due to subsequent injuries, recovery setbacks, dependence, and mortality. A growing body of dual-task (DT) studies suggests a role of executive functions in gait control and falls, particularly in subacute stroke. However, few studies have compared distinct executive and non-executive tasks, nor their effects on chronic stroke gait. Research question: The purpose of this crosssectional study was to compare the effects of distinct working memory (2-back) and inhibition (Stroop) tasks on walking gait performance in chronic stroke survivors.

Methods: A pilot sample of chronic stroke survivors $(n=11,8$ males, mean age $=70.91$, 6-12months poststroke event) and age-matched healthy controls $(n=13,4$ male; mean age $=68.46$ ) were tested. Gait performance (speed, stride time, stride time variability, stride length and stride length variability) was measured using 2 wireless inertial measurement sensors under 4 walking conditions: 1) preferred walking (single-task: ST), 2) walking with a 2-back DT, 3) walking with a Stroop DT, and 4) walking with a non-executive motor response DT. The secondary tasks were also carried out in both ST (seated) and DT conditions, to examine bidirectional effects.

Results: While the stroke survivor sample had a slower gait speed across conditions and tasks, there were no significant differences between the groups $\left[F(1,22)=1.13, p=.299, \eta^{2} p=.049\right]$ on the spatial or temporal gait characteristics recorded: gait performance was maintained during executive and non-executive DTs. In addition, we did not find a significant effect of group on cognitive task performance (all $p>.052$ ). However, we observed a cost in accuracy on the 2-back DT for both groups, suggesting resource overlap and greater cognitive load (all $t>19.72$, all $p<.001$ ).

Significance: Our gait data contradict previous studies evidencing impaired gait post-stroke, suggesting functional recovery in this chronic stroke sample.
\end{abstract}

Falls are a common problem for stroke survivors in both acute and chronic stages of recovery that can result in multiple negative sequelae: severe setbacks in cognitive and motor recovery, additional injuries, increased hospital stays, loss of independence, and even mortality $[1,2]$. Falls are largely attributed to balance and gait impairments poststroke [1], with gait patterns most often characterised by a slower gait speed, and sometimes with alterations in stride length and cadence that can return to baseline with longer recovery [3,4]. Despite this, fall rates remain higher in long-term stroke survivors than in communitydwelling older adults [5], and cognitive dual-task gait (walking while completing a secondary cognitive task) does not appear to improve over time post-stroke [6]. Indeed, the dual-task literature increasingly suggests a role of cognitive capacity in gait control and falls post-stroke [7].

The dual-task (DT) paradigm allows for the examination of the role of cognitive capacities in gait control (i.e. cognitive-motor interference), by examining the "cost" or change in gait performance from single-task to DT conditions. DT gait post-stroke is most often characterised by slower gait speed, with some studies reporting altered stride length and interference on performance of the secondary

\footnotetext{
* Corresponding author at: Annenberg Public Policy Center, University of Pennsylvania, 202 S 36th Street, Philadelphia, PA, 19104, USA.

E-mail address: elizabeth.walshe@appc.upenn.edu (E.A. Walshe).
} 
cognitive task also (particularly during more challenging walking tasks) [7-10]. The resource capacity theories of attention would suggest that these DT costs are a result of shared cognitive resources being redirected from gait control to the secondary task [11]. In support of this, numerous studies have shown that DT costs in gait performance appear to be greater with higher-level cognitive tasks (compared to motor tasks), amplified in older adults compared to younger adults, and even more pronounced with mild cognitive impairment, dementia and poststroke [12-15]. While subtle cognitive impairment has been documented in subacute stroke [see 16], few studies have examined this over a longer recovery period [17], nor how it may relate to gait impairments and falls in chronic stroke.

A number of studies have examined the impact of cognitive tasks on DT gait post-stroke, but the role of specific cognitive capacities remains unclear, in part, due to conflicting findings [7]. Inconsistent findings can be attributed to a number of problems in the DT literature including a lack of comparative control groups (e.g [8,9,15]), inconsistent analysis of bidirectional effects (on gait and cognitive DT performance), and the heterogeneity of stroke [6]. In addition, there has also been a lack of comparative control dual-tasks in studies that attempted to compare cognitive domains. However, the few studies that have explicitly compared different dual-tasks indeed suggest that the type of task matters. For example, one study found that a spontaneous speech task affected gait performance more than a working memory/updating task or visuospatial decision-making task in a sample of 13 community stroke survivors (but this finding is limited by a lack of a control group) [8]. Another study comparing 10 stroke survivors to healthy young adults (rather than age-matched) found that a visuomotor task interfered most with cognitive performance in both groups, but that the serial subtraction task (working memory/updating) had a greater effect on gait performance in stroke survivors than a Stroop (inhibition/interference) or a visuomotor task [16].

Overall, executive tasks appear to be key in the control of gait post stroke [17]. However, executive function is a complex domain comprised of a number of processes, with various cortical inputs [12,13] that are targeted by distinct and varied tasks. The current study attempts to address some of the knowledge gaps and methodological limitations in the DT literature by comparing the bidirectional interference effects of distinct executive and non-executive dual-tasks, in a pilot sample of stroke survivors and age-matched healthy older adults. Specifically, the executive tasks targeted working memory and cognitive inhibition: two capacities that are impaired post-stroke [18]. We hypothesize that the stroke survivor group will have slower gait speed at baseline and increased DT costs on speed for the executive tasks. We also predict that the executive tasks will elicit greater interference than a control task (non-executive motor response task).

\section{Methods}

\subsection{Participants}

A relatively homogeneous sample of 12 community-dwelling ischaemic stroke survivors (SS) was recruited from Tallaght University Hospital Stroke Unit records. One participant was later excluded due to inability to walk $15 \mathrm{~m}$, leaving 11 SS participants ( 8 male; mean age $=70.91$; mean time post-stroke: 305.29 days). All SS participants had a Modified Rankin Score of less than 2. An age-matched control group of healthy older adults (OA) was also recruited from the community $(n=13,4$ male; mean age $=68.46$ ). The inclusion criteria for both groups included the ability to walk upright for at least $15 \mathrm{~m}$, age 55 years and above, normal or corrected vision and hearing. In addition, we only recruited SS participants that were at least 6 months poststroke, allowing for maximal motor recovery [19]. Participants were excluded if they reported taking medications stating side effects of dizziness or balance impairments. Individuals who were taking numerous medications were often excluded due to other health issues that met the criteria for exclusion: e.g. dementia, aphasia, relevant vestibular or musculoskeletal conditions. Only 2 participants in each group reported a fall in the previous 12 months (thus, we could not compare fallers and non-fallers). This study was conducted in accordance with the ethical standards of the American Psychological Association (APA), and with approval from the Ethical Committees of Maynooth University and Tallaght University Hospital.

\subsection{Measures}

The two groups were compared on measures of height $(\mathrm{cm})$, weight $(\mathrm{kg})$, lower limb strength (5 times sit-to-stand task), fear of falling (FESI [20]), premorbid intelligence (National Adult Reading Test, NART [21]), and global cognition (Mini Mental State Examination and the Montreal Cognitive Assessment [22,23]). Three stimulus-response secondary tasks were utilized (motor, n-back and Stroop task): all generated in E-Prime and run on a Dell Latitude $2.1 \mathrm{GHz}$ Intel Pentium Processor laptop. Auditory stimuli (mono 16-bit sound files with a $44.1 \mathrm{kHz}$ sampling rate) were presented via wireless fm headphones, and participants responded as instructed on a hand-held wireless mouse. Accuracy (ACC: \%) and response time (RT: ms) were the key outcome measures. Both the gait and secondary tasks were examined in single-task (ST) and DT conditions (tasks and conditions were counterbalanced across participants). No instruction was given regarding which task to prioritise in the DT condition. Dual-task change (DTC) was derived from the change in performance from single- to dual-task conditions. Testing took place at Tallaght Hospital and Maynooth University, using a $15 \mathrm{~m}$ corridor for the walking trials, and a private testing room for cognitive and other assessments.

\subsubsection{Secondary tasks}

Motor Task. The motor task was a basic stimulus-response control task that requires attention but does not tax higher-level executive function or decision-making processes. A single auditory tone was presented $(1000 \mathrm{~ms}$ long with a $1200 \mathrm{~ms}$ response window from stimulus onset) at randomly varied delay intervals $(500 \mathrm{~ms}, 750 \mathrm{~ms}$ or $1000 \mathrm{~ms}$ ), to which participants were instructed to quickly respond by clicking the left button of the mouse.

2-Back Task. An auditory 2-back task was employed to assess executive working memory [24]. The participants heard a sequence of nouns (Toronto Noun Pool), presented one at a time, and were asked to respond by clicking the left mouse button when the current word was a repeat of the word presented two trials previously. The response window was $2000 \mathrm{~ms}$ in length from the time of stimulus onset. A short practice block was given before the test block began. Target "match" appeared on $30 \%$ of trials.

Stroop Task. An auditory response conflict Stroop task was utilised to target inhibition [25]. Again, a short practice block was presented before the test block. There were 4 auditory voice stimuli consisting of the words "High" and "Low" played in either a high (Hi: $300 \mathrm{~Hz}$ ) or low (Lo: $160 \mathrm{~Hz}$ ) pitch, presented with a $2000 \mathrm{~ms}$ response window and a $300 \mathrm{~ms}$ inter-trial-interval. Each of the four stimuli occurred on $25 \%$ of the trials in a pseudorandom order to ensure no more than 2 consecutive repetitions, with incongruent stimuli appearing on $50 \%$ of trials. There were two response options: a left mouse button click for congruent stimuli ("High" ${ }_{\mathrm{Hi}}$ and "Low" ${ }_{\mathrm{LO}}$ ) and a right mouse button click for incongruent stimuli ("High" ${ }_{\mathrm{Lo}}$ and "Low" ${ }_{\mathrm{Hi}}$ ). Congruent and incongruent ACC and RT (respectively) were measured.

\subsubsection{Gait assessment}

Participants completed 2 single-task (ST) walking trials (to get an average of ST gait), and 3 dual-task (DT) walking trials. Tasks and trial order were counterbalanced. Each trial consisted of walking at a preferred speed along a straight, wide, well-lit, and obstacle-free $15 \mathrm{~m}$ walkway with an about-turn at each end (mapped out with floor markings). Two wireless inertial measurement sensors (SHIMMER2R: 
Shimmer Research LTD, Dublin, Ireland) were secured to the shank of the left and right leg, and recorded tri-axial accelerometer and gyroscope data at a sampling rate of $102.4 \mathrm{~Hz}$. A previously validated algorithm developed in MATLAB $^{\circledR}$ was used to identify gait events in the sagittal plane gyroscope signal and calculate temporal and spatial gait metrics from these events [26]. Five gait variables were analysed: gait speed $(\mathrm{m} / \mathrm{s})$; stride time $(\mathrm{s})$; stride time variability (Coefficient of Variability \%: CV stride time); stride length (m); and stride length variability (CV stride length \%).

\subsection{Statistical analysis}

All statistical analyses were carried out using SPSS 21 statistical package (SPSS Software, Seattle, WA, USA). Group comparisons on control measures were conducted using independent t-tests. Mixed factorial ANOVAs were used to analyze between- and within-group condition effects on cognitive and gait outcomes, and DTC values. Bonferroni corrected follow-up t-tests were used to examine simple main effects.

\section{Results}

\subsection{Group comparisons}

There were no significant differences between the OA and SS groups on the general control measures (all $\mathrm{p}>$.462): see Table 1. FES-I scores indicated moderate concern about balance and falling in both groups [27].

\subsection{Gait analysis}

The SS group had slower gait speeds on all ST and DT trials compared to the OA group (see Table 2), but there were no statistically significant effects of Group $\left[\left[F(1,22)=1.13, p=.299, \eta^{2} p=.049\right]\right.$ or task $\left[F(3,66)=0.50, p=.684, \eta^{2} p=.022\right]$. Analysis of the other gait variables revealed no significant main effects of Group or Task Type (ST, Motor DT, n-back DT or Stroop DT): all $F<3.62$, all $p>.072$.

\subsubsection{Gait dual-task change}

Dual-Task Change (DTC; \%) values for each of the gait variables were examined: see Table 3. There were no main effects of Task Type or Group on speed DTC, CV stride time DTC, mean stride length DTC, or CV stride length DTC: All F $<3.04$, all $\mathrm{p}>$.059. For mean stride time DTC, there was a significant main effect of Task $[F(2,40)=6.30, p$ $\left.=.004, \eta^{2} p=.239\right]$ but no main effect of Group $[F(1,20)=2.15, p$ $\left.=.158, \eta^{2} p=.097\right]$. However, follow-up analysis using the Bonferroni correction found no significant differences between the groups (all $t<1.77$, all $p>$.09), or across the 3 dual-tasks (all $t<2.38$, all $p>$ 0.036: corrected $\alpha=.013$ ).

Table 1

Mean (and standard error of the mean) values for control variables in the older adult (OA) and stroke survivors (SS) participant groups, with p values for comparisons between groups.

\begin{tabular}{llll}
\hline Measure & OA & SS & $\mathrm{p}$ \\
\hline Age (years) & $68.46(2.35)$ & $70.91(2.22)$ & 0.462 \\
Height (cm) & $166.73(1.79)$ & $166.05(3.20)$ & 0.849 \\
Weight (kg) & $70.51(3.97)$ & $74.09(3.06)$ & 0.496 \\
5 Times Sit-to-Stand (s) & $10.25(0.62)$ & $11.03(1.05)$ & 0.511 \\
NART Full Scale IQ & $109.8(2.63)$ & $109.27(2.27)$ & 0.858 \\
FES-I & $23.46(1.67)$ & $23.45(1.67)$ & 0.998 \\
MMSE $^{\circledR}$ & $27.92(0.60)$ & $28.45(0.65)$ & 0.556 \\
MoCA & $24.46(1.17)$ & $23.55(1.12)$ & 0.582 \\
\hline
\end{tabular}

\subsection{Cognitive analysis}

No significant between-group differences were found on any of the secondary tasks (all $t<2.05$, all $p>$.052). Within-group comparisons showed that both the OA and SS group were less accurate on the DT 2back in comparison to the ST (non-walking) condition (see Fig. 1a): all $t>19.72$, all $p<.001$. The OA group also had faster RTs when responding correctly on the DT 2-back in comparison to the ST (Fig. 1b): $t$ $(11)=3.34, p=.007$. The same RT trend was observed in the SS group, but the effect was not significant $(p=.08)$.

\subsubsection{Cognitive dual task change}

Separate comparisons of RT DTC and ACC DTC values were made between and within groups across tasks. There were no differences between the two groups on accuracy DTC values (all $\mathrm{t}<.316$, all $\mathrm{p}>$.108) or RT DTC values (all $\mathrm{t}<1.96$, all $\mathrm{p}>$.064). Comparing across tasks within the OA group, there was a greater ACC DTC on the 2-back $(M=41.05, S E M=1.88)$, than on incongruent Stroop trials ( $M$ $=-12.55, \operatorname{SEM}=10.02)[\mathrm{t}(10)=5.10, \mathrm{p}<.001]$. This finding is likely due to the decline in accuracy on the 2-back in the DT condition, and parallel increase in accuracy on incongruent trials in the Stroop DT. There was also a significant difference in RT DTC between the 2-back $(M=-20.43, S E M=5.61)$ and congruent Stroop trials $(M=11.71$, $\mathrm{SEM}=6.67)$ in the OA group: $\mathrm{t}(10)=-3.69, \mathrm{p}=.004$. This is due to their faster RTs on the 2-back DT (DT improvement) compared to slower RTs on the congruent Stroop trials (DT cost).

Within the SS group, there was a significant difference between the increased RTs (DT cost) on the Motor DT and the decreased RTs on the 2-back DT $[\mathrm{t}(9)=4.13, \mathrm{p}=.003]$ and congruent Stroop DT [t $(10)=3.61, p=.005]$. See Fig. 2 for DTC in ACC and RT performances.

\section{Discussion}

This study examined the relative role of distinct executive function processes in the control of gait post-stroke, by comparing the interference effects of a working memory and an inhibitory control DT on performance in stroke survivors and age-matched healthy older adults. Surprisingly, we found no significant differences between the groups on any of the spatial or temporal gait variables recorded at baseline, nor in the dual-task condition. However, we did see a trend that our stroke survivor sample had a slower gait overall. Although not statistically significant, this trend is in line with prior studies which commonly report reduced speed as characteristic of post-stroke gait [3]. In contrast, prior work has shown that other impaired gait characteristics such as decreased stride length and cadence post-stroke tend to recover over a longer period of time post-rehabilitation [3,4]. This may explain why we did not find group differences on these metrics in our stroke survivors sample who were more than 10 months post-stroke event. More challenging walking tasks may reveal group differences [10]. However, one study also found that neither stroke nor healthy adults were impaired in obstacle avoidance while concurrently completing a Stroop task [28].

There was no significant decline in gait in the DT condition, for any of the secondary tasks (nor any patterns of differences in relative DTC between the tasks). For the cognitive measures, we did not see baseline impairments in executive function capacities in this stroke survivor sample. However, we did see costs in cognitive performance on the working memory DT for both groups, which indicates overlapping resources between working memory and motor processing, and highlights the importance of examining bidirectional effects in DT studies. We also saw increased accuracy on incongruent trials in controls, and longer RTs in the stroke survivors. However, accuracy scores were close to $50 \%$ on both the ST and DT conditions in the stroke survivor group (and to a lesser extent in the control group). This may indicate that stroke survivors responded at chance when unsure of the correct response, 
Table 2

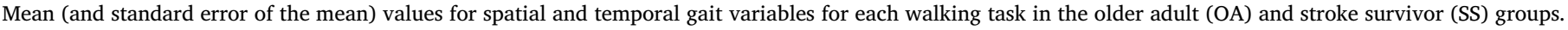

\begin{tabular}{|c|c|c|c|c|c|c|c|c|}
\hline \multirow[b]{2}{*}{ Gait Measure } & \multicolumn{4}{|l|}{$\mathrm{OA}$} & \multicolumn{4}{|l|}{ SS } \\
\hline & ST & Motor DT & 2-back DT & Stroop DT & ST & Motor DT & 2-back DT & Stroop DT \\
\hline Speed (m/s) & $1.20(0.03)$ & $1.17(0.04)$ & $1.20(0.03)$ & $1.16(0.03)$ & $1.14(0.03)$ & $1.15(0.04)$ & $1.14(0.04)$ & $1.13(0.04)$ \\
\hline Stride Time (s) & $0.93(0.02)$ & $0.94(0.02)$ & $0.96(0.02)$ & $0.97(0.02)$ & $0.97(0.02)$ & $0.97(0.03)$ & $0.98(0.03)$ & $0.96(0.02)$ \\
\hline CV Stride Time (\%) & $7.28(0.59)$ & $7.63(0.71)$ & $6.36(0.52)$ & $8.10(1.10)$ & $6.28(0.26)$ & $6.13(0.21)$ & $5.85(0.23)$ & $6.26(0.19)$ \\
\hline Stride Length (m) & $1.11(0.01)$ & $1.09(0.03)$ & $1.15(0.01)$ & $1.12(0.02)$ & $1.10(0.02)$ & $1.11(0.02)$ & $1.11(0.02)$ & $1.11(0.02)$ \\
\hline CV Stride Length (\%) & $48.06(0.91)$ & $46.26(2.05)$ & $45.38(1.14)$ & $47.47(1.63)$ & $48.34(1.01)$ & $48.82(1.72)$ & $47.29(1.26)$ & $48.06(1.10)$ \\
\hline
\end{tabular}

Table 3

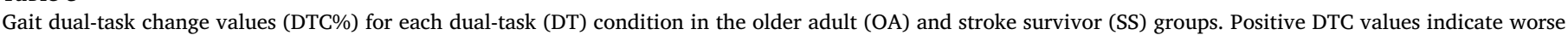
performance on the DT than ST (i.e. DT costs).

\begin{tabular}{|c|c|c|c|c|c|c|}
\hline \multirow[b]{2}{*}{ Gait DTC } & \multicolumn{3}{|l|}{ OA } & \multicolumn{3}{|l|}{ SS } \\
\hline & Motor DT & 2-back DT & Stroop DT & Motor DT & 2-back DT & Stroop DT \\
\hline Speed DTC\% & $2.06(3.47)$ & $-0.73(2.98)$ & $2.34(3.24)$ & $-1.44(2.25)$ & $-0.58(2.07)$ & $0.81(2.31)$ \\
\hline Stride Time DTC\% & 1.09 (1.93) & $3.57(1.59)$ & $2.64(1.42)$ & $-0.94(1.19)$ & $-0.04(1.18)$ & $-0.11(1.04)$ \\
\hline CV Stride Time DTC\% & $17.32(12.02)$ & $-4.87(4.15)$ & $11.43(9.41)$ & $-7.64(4.55)$ & $-8.77(3.01)$ & $0.26(3.85)$ \\
\hline Stride Length DTC\% & $1.43(2.87)$ & $-3.91(2.20)$ & $-1.08(2.46)$ & $-0.37(1.74)$ & $-0.44(1.64)$ & $-1.15(2.08)$ \\
\hline CV Stride Length DTC\% & $3.25(5.33)$ & $-5.11(3.19)$ & $-0.65(4.30)$ & $1.11(3.32)$ & $-1.72(3.45)$ & $-0.05(3.40)$ \\
\hline
\end{tabular}
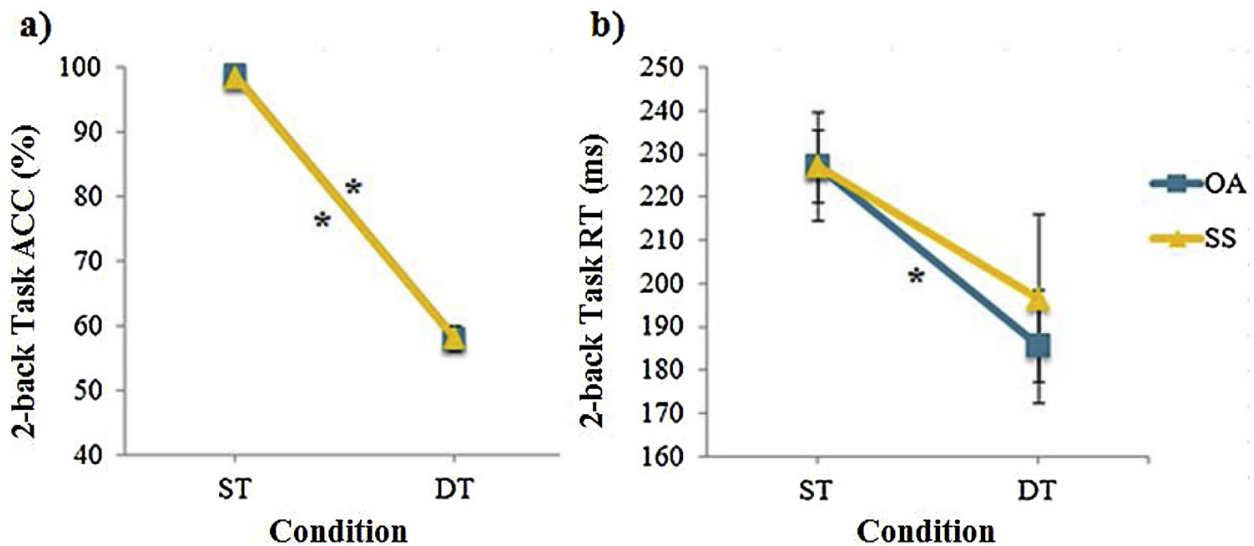

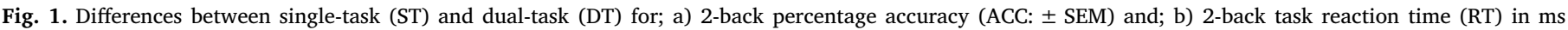
( \pm SEM), in both the older adult (OA) and stroke survivor (SS) groups (* indicates significance at the Bonferroni adjusted alpha).

which could also allow for prioritising maintenance of walking task performance. Overall, these findings are in contrast to previous work that has shown cognitive-motor interference on speed, stride time, and cognitive performance post-stroke [6-9]. However, our working memory n-back task differed from previous studies using the serial serial-subtraction [14]. Furthermore, few studies have examined cognitive impairment and DT gait specifically in chronic stroke. Thus, these findings may suggest that there is substantial functional recovery in the chronic stage of stroke (230-438 days post-stroke).

Interestingly, our stroke survivor sample had a faster baseline mean gait speed than previously reported speeds for even high-functioning survivors [29], and our older adult group also had a slower mean speed than national reference data (see [30]). Furthermore, this OA group had relatively low scores on the MMSE and MoCA (scoring in the 25th percentile or lower relative to national reference data: [30]). This suggests that we recruited a slightly poorer-performing healthy control group and a particularly well-recovered stroke survivor group, which may account for the equivalence between our groups on the control measures (IQ, strength, etc.). These results support the hypothesis that time in stroke recovery (stroke chronicity) is a critical factor moderating the relationship between cognitive and motor control in dual-task gait [9]. Stroke survivors who have had more time in recovery poststroke may demonstrate less cognitive-motor interference due to extended motor recovery and reacquisition of gait automaticity. Further research should attempt to replicate these findings, with larger sample sizes, and also consider comparing fallers and non-fallers post-stroke, as they may evidence different cognitive-motor impairments.

\subsection{Limitations}

The small sample size of this study, with a conservative Bonferroni correction for multiple post-hoc comparisons, may have limited the ability to detect group and task differences. However, previous studies with similar designs were able to detect main effects in similar sized samples $[9,14]$. Second, self-selection bias for study participation may have resulted in a well-recovered stroke survivor sample that does not represent the general chronic stroke population. Third, task selection and difficulty may not have been appropriate for revealing cognitive impairments and overlapping resources in DT gait. Additionally, a variable inter-stimulus interval should have been used to avoid any positive effects of stimulus timing on gait rhythmicity.

\subsection{Conclusion}

Overall, the data did not support our predictions for group and task differences. Our findings suggest that despite the damage incurred by a 


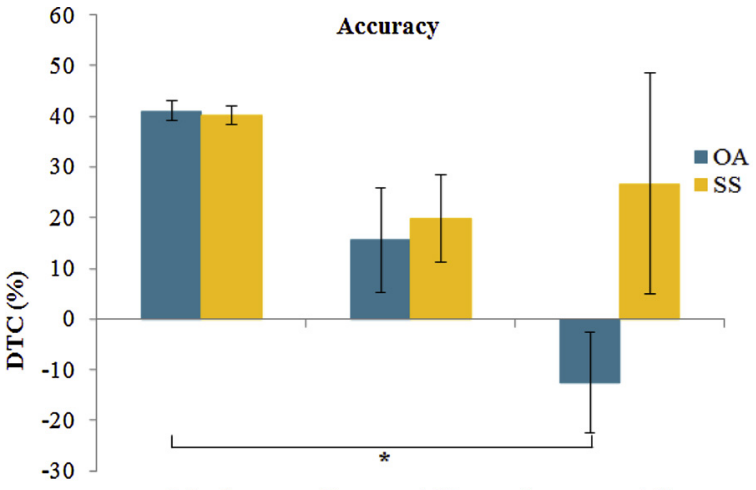

2-back Congruent Stroop Incongruent Stroop

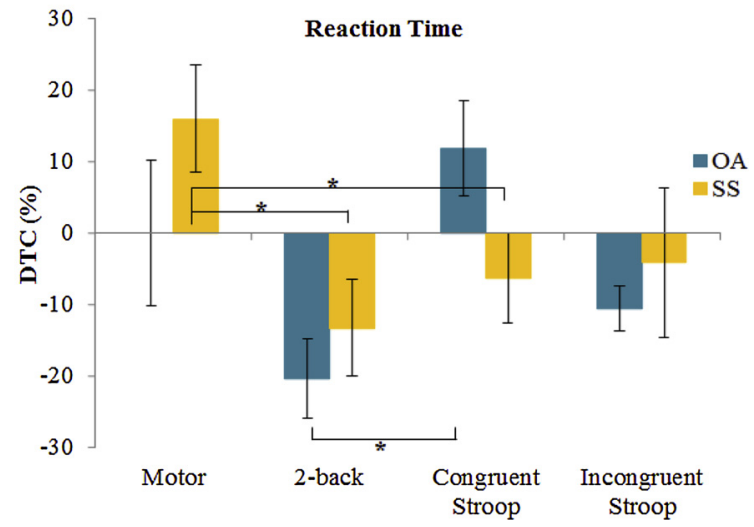

Fig. 2. Mean dual-task change (DTC\%) in; a) accuracy ( \pm SEM); and b) reaction time ( \pm SEM), for each dual-task in both the older adult (OA) and stroke survivor (SS) groups (* indicates significance at the Bonferroni adjusted alpha).

stroke, time in recovery is critical: survivors ( $~ 300$ days post-stroke) can return to performance levels equivalent to age-matched (but potentially poor-performing) controls, and thus, normative functioning life. It is important to remember that impairment and recovery post-stroke depends on a multitude of factors, as do falls. Thus, stroke survivors should not be treated as a homogeneous group when considering impairments or intervention. However, this study was not without limitations. Future research should continue to compare the effects of different cognitive DTs on gait control at different stages of stroke recovery, particularly among fallers and non-fallers.

\section{Conflict of interest statement}

The authors declare that the research was conducted in the absence of any commercial or financial relationships that could be construed as a potential conflict of interest.

\section{Acknowledgements}

The authors would like to acknowledge support from the Irish Research Council and Irish Heart Foundation, and also thank Joe Duffin at Maynooth University for valuable discussion.

\section{References}

[1] F.A. Batchelor, S.F. Mackintosh, C.M. Said, K.D. Hill, Falls after stroke, Int. J. Stroke 7 (6) (2012) 482-490, https://doi.org/10.1111/j.1747-4949.2012.00796.x.

[2] J.S. Wong, D. Brooks, E.L. Inness, A. Mansfield, The impact of falls on motor and cognitive recovery after discharge from in-patient stroke rehabilitation, J. Stroke Cerebrovasc. Dis. 25 (7) (2016) 1613-1621, https://doi.org/10.1016/j. jstrokecerebrovasdis.2016.03.017.

[3] J. Jonsdottir, M. Recalcati, M. Rabuffetti, A. Casiraghi, S. Boccardi, M. Ferrarin, Functional resources to increase gait speed in people with stroke: strategies adopted compared to healthy controls, Gait Posture 29 (3) (2009) 355-359, https://doi.org/ 10.1016/j.gaitpost.2009.01.008.

[4] K.K. Patterson, I. Parafianowicz, C.J. Danells, V. Closson, M.C. Verrier, W.R. Staines, et al., Gait asymmetry in community-ambulating stroke survivors, Arch. Phys. Med. Rehabil. 89 (2) (2008) 304-310, https://doi.org/10.1016/j.apmr. 2007.08.142.

[5] L. Jorgensen, T. Engstad, B.K. Jacobsen, Higher incidence of falls in long-term stroke survivors than in population controls: depressive symptoms predict falls after stroke, Stroke 33 (2) (2002), https://doi.org/10.1161/hs0202.102375.

[6] D. Hyndman, A. Ashburn, L. Yardley, E. Stack, Interference between balance, gait and cognitive task performance among people with stroke living in the community, Disabil. Rehabil. 28 (13-14) (2006) 849-856, https://doi.org/10.1080/ 09638280500534994.

[7] P. Plummer, G. Eskes, S. Wallace, C. Giuffrida, M. Fraas, G. Campbell, et al., Cognitive-motor interference during functional mobility after stroke: state of the science and implications for future research, Arch. Phys. Med. Rehabil. 94 (12) (2013) 2565-2574, https://doi.org/10.1016/j.apmr.2013.08.002 e6.

[8] P. Plummer-D'Amato, L.J.P. Altmann, D. Saracino, E. Fox, A.L. Behrman, M. Marsiske, Interactions between cognitive tasks and gait after stroke: a dual task study, Gait Posture 27 (4) (2008) 683-688, https://doi.org/10.1016/j.gaitpost. 2007.09.001.

[9] J. Shin, H. Choi, J.A. Lee, S. Eun, D. Koo, J. Kim, S. Lee, K. Cho, Dual task interference while walking in chronic stroke survivors, Phys. Ther. Rehabil. Sci. 6 (2017) 134-139, https://doi.org/10.14474/ptrs.2017.6.3.134.

[10] C. Timmermans, M. Roerdink, T.W.J. Janssen, C.G.M. Meskers, P.J. Beek, Dual-task walking in challenging environments in people with stroke: cognitive-motor interference and task prioritization, Stroke Res. Treat. (2018) 7928597, , https://doi org/10.1155/2018/7928597.

[11] H.E. Pashler, J.C. Johnston, Attentional limitations in dual-task performance, Attention (1998) 155-189, https://doi.org/10.1016/j.tics.2005.01.008 Hove, England Psychology.

[12] E.A. Walshe, M.R. Patterson, S. Commins, R.A.P. Roche, Dual-task and electrophysiological markers of executive cognitive processing in older adult gait and fallrisk, Front. Hum. Neurosci. 9 (2015) 200, https://doi.org/10.3389/fnhum.2015. 00200.

[13] G. Yogev-Seligmann, J.M. Hausdorff, N. Giladi, The role of executive function and attention in gait, Mov. Disord. 23 (3) (2008) 329-342, https://doi.org/10.1002/ mds. 21720.

[14] P. Patel, T. Bhatt, Task Matters: influence of different cognitive tasks on cognitivemotor interference during dual-task walking in chronic stroke survivors, Top. Stroke Rehabil. 21 (4) (2014) 347-357, https://doi.org/10.1310/tsr2104-347.

[15] H. Manaf, M. Justine, G.H. Ting, L.A. Latiff, Comparison of gait parameters across three attentional loading conditions during timed up and go test in stroke survivors, Top. Stroke Rehabil. 21 (2) (2014) 128-136, https://doi.org/10.1310/tsr2102-128.

[16] J.T. Duffin, D.R. Collins, T. Coughlan, D. O'Neill, R.A.P. Roche, S. Commins, Subtle memory and attentional deficits revealed in an Irish stroke patient sample using domain-specific cognitive tasks, J. Clin. Exp. Neuropsychol. 34 (8) (2012) 864-875, https://doi.org/10.1080/13803395.2012.690368.

[17] S. Verstraeten, R. Mark, M. Sitskoorn, Motor and cognitive impairment after stroke: a common bond or a simultaneous deficit? Stroke Res Ther. 1 (1) (2016).

[18] T.B. Cumming, R.S. Marshall, R.M. Lazar, Stroke, cognitive deficits, and rehabilitation: still an incomplete picture, Int. J. Stroke 8 (1) (2013) 38-45, https:// doi.org/10.1111/j.1747-4949.2012.00972.x.

[19] P.W. Duncan, L.B. Goldstein, D. Matchar, G.W. Divine, J. Feussner, Measurement of motor recovery after stroke, Stroke 23 (1992) 1084-1089.

[20] L. Yardley, N. Beyer, K. Hauer, G. Kempen, C. Piot-Ziegler, C. Todd, Development and initial validation of the Falls Efficacy Scale-International (FES-I), Age Ageing 34 (6) (2005) 614-619, https://doi.org/10.1093/ageing/afi196.

[21] H.E. Nelson, The National Adult Reading Test (NART): Test Manual, 124(3) NFERNelson, Windsor, UK, 1982, pp. 0-25 http://doi.org/Thesis_references-Converted\# 319.

[22] D. Molloy, E. Alemayehu, R. Robert, A standardized mini-mental state examination: its reliability compared to the traditional mini-mental state examination, Am. J. Psychiatry 48 (1991) 102-105, https://doi.org/10.1176/ajp.148.1.102.

[23] Z.S. Nasreddine, N.A. Phillips, V. Bédirian, S. Charbonneau, V. Whitehead, I. Collin, et al., The Montreal Cognitive Assessment, MoCA: A brief screening tool for mild cognitive impairment, J. Am. Geriatr. Soc. 53 (4) (2005) 695-699, https://doi.org/ 10.1111/j.1532-5415.2005.53221.x.

[24] A.M. Owen, K.M. McMillan, A.R. Laird, E. Bullmore, N-Back working memory paradigm: a meta-analysis of normative functional neuroimaging studies, Hum. Brain Mapp. 25 (2005) 46-59, https://doi.org/10.1002/hbm.20131.

[25] R.E. Shor, An auditory analog of the Stroop Test, J. Gen. Psychol. 93 (2d Half) (1975) 281-288.

[26] M.R. Patterson, E. Delahunt, K.T. Sweeney, B. Caulfield, An ambulatory method of identifying anterior cruciate ligament reconstructed gait patterns, Sensors (Basel, Switzerland) 14 (2014) 887-899, https://doi.org/10.3390/s140100887.

[27] K. Delbaere, J.C.T. Close, A.S. Mikolaizak, P.S. Sachdev, H. Brodaty, S.R. Lord, The falls efficacy scale international (FES-I), A comprehensive longitudinal validation study, Age Ageing 39 (2) (2010) 210-216, https://doi.org/10.1093/ageing/afp225.

[28] K. Smulders, R. van Swigchem, B.J.M. de Swart, A.C.H. Geurts, V. Weerdesteyn, Community-dwelling people with chronic stroke need disproportionate attention while walking and negotiating obstacles, Gait Posture 36 (1) (2012) 127-132, https://doi.org/10.1016/j.gaitpost.2012.02.002.

[29] C. Beyaert, R. Vasa, G.E. Frykberg, Gait post-stroke: pathophysiology and rehabilitation strategies, Neurophysiol. Clin. Neurophysiol. 45 (4-5) (2015) 335-355, https://doi.org/10.1016/j.neucli.2015.09.005.

[30] R.A. Kenny, R.F. Coen, J. Frewen, O.A. Donoghue, H. Cronin, G.M. Savva, Normative values of cognitive and physical function in older adults: findings from the Irish Longitudinal Study on Ageing, J. Am. Geriatr. Soc. 61 (2013) S279-S290, https://doi.org/10.1111/jgs.12195. 